\title{
A Method for Preservation and Determination of welding fume Nanoparticles in Exhaled Breath Condensate
}

Halshka Graczyk ${ }^{1^{*}}$, Sabrina Gschwind ${ }^{2 *}$, Detlef Günther ${ }^{2}$, Michael Riediker ${ }^{1,3}$

${ }^{1}$ University of Lausanne and Geneva, Institute for Work and Health, Route de la Corniche 2, CH-1066

Epalinges-Lausanne, Switzerland.

${ }^{2}$ ETH Zurich, Department of Chemistry and Applied Biosciences, Laboratory of Inorganic Chemistry, Vladimir-Prelog-Weg 1, CH-8093 Zurich, Switzerland

${ }^{3}$ IOM Singapore, 30 Raffles Place \#17-00, Singapore 048622, Singapore

*Contributed equally

Email: Halshka.Graczyk@chuv.ch

Analysis of exhaled breath condensate (EBC) represents a non-invasive method for detecting inhaled nanoparticles (NP) associated with various occupational and environmental exposures. However, the few studies that have investigated NPs in EBC often assess only bulk, ionic intensities to provide information on overall elemental content, rather than on particulate content. In an attempt to assess inhaled metallic particles in their original particulate form, two EBC preservation strategies were tested: either flash freezing EBC immediately after collection, or keeping EBC at room temperature (RT) until analysis. Particle content and their elemental composition of the differently preserved samples were analyzed by Microdroplet Generation Inductively Coupled Plasma Mass Spectrometry and by Electron Microscopy. $\mathrm{Mn}, \mathrm{Fe}, \mathrm{Al}, \mathrm{Ni}$ and $\mathrm{Sn}$ were present in the samples in different form depending on storage strategy. The sample stored at RT showed significant levels of ionic $\mathrm{Al}$ and $\mathrm{Ni}$, whereas the other analyzed elements were found in particulate form. In the flash frozen sample defrosted shortly before analysis, all metals were detected only in particulate form. Our findings indicate that welding fume NPs in EBC may quickly and uncontrollably degrade, losing their original form and hampering accurate characterization analysis. We demonstrate the importance of flash freezing EBC samples immediately after collection and defrosting them shortly before analysis at a temperature that does not affect proteins and peptides $\left(<38^{\circ} \mathrm{C}\right)$ in order to effectively preserve NPs in particulate form. This methodology can be applied easily, effectively, and inexpensively, especially in field studies, to preserve EBC samples for future NP characterization. 